\title{
Effects of Bleaching Associated with Er:YAG and Nd:YAG Laser on Enamel Structure and Bacterial Biofilm Formation
}

\author{
Xiuxiu Hou $\mathbb{D}^{1,2,3,4,5}$ Keyong Yuan, ${ }^{1,2,3,4,5}$ Zhengwei Huang, ${ }^{1,2,3,4,5}$ and Rui Ma $\mathbb{D}^{1,2,3,4,5}$ \\ ${ }^{1}$ Department of Endodontics, Shanghai Ninth People's Hospital, Shanghai Jiao Tong University School of Medicine, \\ Shanghai 200011, China \\ ${ }^{2}$ College of Stomatology, Shanghai Jiao Tong University, Shanghai 200011, China \\ ${ }^{3}$ National Center for Stomatology, Shanghai 200011, China \\ ${ }^{4}$ National Clinical Research Center for Oral Diseases, Shanghai 200011, China \\ ${ }^{5}$ Shanghai Key Laboratory of Stomatology, Shanghai 200011, China
}

Correspondence should be addressed to Rui Ma; marui1723@sina.com

Received 4 October 2021; Accepted 3 December 2021; Published 20 December 2021

Academic Editor: Lavinia C. Ardelean

Copyright (C) 2021 Xiuxiu Hou et al. This is an open access article distributed under the Creative Commons Attribution License, which permits unrestricted use, distribution, and reproduction in any medium, provided the original work is properly cited.

\begin{abstract}
Objective. To compare the effects of bleaching associated with Er:YAG and Nd:YAG laser on enamel structure and mixed biofilm formation on teeth surfaces. Materials and Methods. Sixty-eight enamel samples were randomly divided into four groups ( $n=17)$, control, Opalescence Boost only, Opalescence Boost plus Er: YAG laser, and Opalescence Boost plus Nd:YAG laser. The structure was observed using SEM after bleaching. Subsequently, the treated enamel samples were also cultured in suspensions of Streptococcus mutans, Streptococcus sanguis, Actinomyces viscosus, and Fusobacterium nucleatum (Fn) for 24 and $48 \mathrm{~h}$. Biofilm formation was quantified by crystal violet staining, and the structure was visualized by confocal laser scanning microscopy. The data were analyzed using the Kruskal-Wallis method. Results. The enamel structure significantly changed after bleaching. There was no obvious difference in the biofilm formation after $24 \mathrm{~h}$; however, after 48 hours, the amount of biofilm increased significantly. Remarkably, the amount was significantly higher on enamel bleached only, however, there was no significant difference between samples bleached with Er:YAG or Nd:YAG laser compared to the control. Conclusions. Bleaching only appeared to markedly promote biofilm formation after $48 \mathrm{~h}$, and the biofilms on samples bleached with Er:YAG or Nd:YAG laser did not change significantly, showing that bleaching with Er:YAG or Nd:YAG laser can be safely applied in clinical practice.
\end{abstract}

\section{Introduction}

Tooth staining is an aesthetic problem that plagues many people. Compared to veneers and crown restorations, tooth bleaching is a conservative and noninvasive option for treating tooth staining [1]. Moreover, in-office bleaching with high concentrations of hydrogen peroxide can meet patients' demands quickly. Recently, it has been accepted that hydrogen peroxide can penetrate the tooth structure and produce free radicals that oxidize the colored organic molecules [2] $[3,4]$. The reaction can also be accelerated under photochemical reactions initiated by light or laser [5].
Laser tooth bleaching began in 1996 with FDA approval of argon $(488 / 514 \mathrm{~nm})$ and carbon dioxide (10600 $\mathrm{nm})$ lasers. In 2007, diode lasers (980 $\mathrm{nm}$ ) also received FDA approval. Recently, photodynamic bleaching has been viewed as an ideal treatment option because of its shorter working time and lower postoperative hypersensitivity [6, 7]. LightWalker dual-wavelength laser from Fotona has two distinct wavelengths, including the Er:YAG (erbium: yttrium-aluminumgarnet) laser $(2940 \mathrm{~nm})$ and the Nd:YAG (neodymium:yttrium-aluminum-garnet) laser (1064 $\mathrm{nm}$ ). The wavelength of Er:YAG laser is close to the water absorption peak, and the energy is mostly absorbed by the gel for heating, which 
reduces the damage to the hard tissue of teeth and renders the procedure safe and minimally invasive [8]. Nd:YAG laser can quickly decompose hydrogen peroxide and penetrate into the tooth to achieve a good bleaching effect.

However, clinical applications of tooth bleaching still impart many adverse effects on soft and hard tissues [9]. Studies have shown that laser-activated bleaching agents seem to be more surface-friendly than other bleaching systems [10], and laser-assisted bleaching using Er,Cr:YSGG lasers did not affect enamel [11]. In contrast, partial loss of the surface enamel can be observed after frequent KTP laser [12], and home-bleached enamel treated with Nd:YAG and Er:YAG laser exhibited some melting and recrystallized areas [13], suggesting that tooth whitening procedures using lasers damage the enamel surface more aggressively than simple peroxide treatment [14]. Such alterations to the enamel surface could also subsequently affect oral bacterial adhesion [15].

Caries is one of the most prevalent infectious diseases worldwide, and the imbalance or dysbiosis of the microbial population within the biofilm covering enamel can lead to caries [16], so it is important to study the formation of biofilms on bleached enamels [17]. At present, the effect of bleaching on the formation of bacterial biofilms is inconclusive, and studies found significant reductions in $S$. mutans populations in subgingival and supragingival plaques after bleaching [18]. However, other studies revealed that bleaching with $35 \%$ hydrogen peroxide markedly promoted $S$. mutans and S. sanguinis biofilm formation [15] [19]. And to our knowledge, no study has examined biofilm formation on enamel after in-office bleaching with Er:YAG or Nd:YAG laser.

As we know, the oral cavity is a complex microecological environment that contains a large number of different bacterial species, proteins, and impurities, it is difficult to replicate the real oral environment via a single kind of bacteria [17], and the biofilm formed by mixed bacteria is more similar to the oral biofilm. Streptococcus mutans is recognized as the most important cariogenic bacterium. Its acid production and acid resistance play important roles in the occurrence and development of caries [20]. Streptococcus sanguis is a pioneering colonizer, aiding in the attachment of subsequent organisms, and is a key bacterium in oral biofilm development [21]. As a smart cariogenic pathogen, Actinomyces viscosus can store polysaccharides and prolong acid production upon sugar deficiency [22]. Fusobacterium nucleatum is one of the most common Gram-negative anaerobic bacteria in dental plaques and can coaggregate with primary colonizing bacteria; thus, playing an important role in the development and maturation of dental plaques [23]. Therefore, this study intended to use the above four bacteria to simulate the formation of oral plaque biofilm.

Thus, the aim of this study was to examine enamel surface structure and biofilm formation of mixed bacteria on human enamel samples after bleaching with or without the use of Er:YAG and Nd:YAG laser. Three null hypotheses were set prior the study; $\mathrm{H}_{0} 1$ : the laser-assisted tooth bleaching treatments would not change the enamel structure compared to the conventional bleaching technique; $\mathrm{H}_{0} 2$ : there would be no differences in biofilm amount among the groups after $24 \mathrm{~h} ; \mathrm{H}_{0} 3$ : there would be no differences in biofilm amount among the groups after $48 \mathrm{~h}$.

\section{Materials and Methods}

2.1. Specimen Preparation and Treatment. Sixty-eight enamel samples were prepared from sound permanent third molars extracted due to periodontal complications or orthodontic treatments. Samples were stored in $0.9 \%$ normal saline. The teeth were polished with pumice and a rubber cup and cut into $4 \mathrm{~mm} \times 4 \mathrm{~mm} \times 1 \mathrm{~mm}$ sections. The upper left corner of the samples was rounded to distinguish the front and the back. Specimens without cracks observed under a stereo microscope (Leica MC170 HD, Germany) were chosen and ground flat with 400-, 600-, 1200-, and 2000 -grit silicon carbide papers. Samples were then randomly divided into four groups of 17 specimens each, including the (A) control, (B) Opalescence Boost only (Opalescence Boost, USA), (C) Opalescence Boost plus Er: YAG laser treatment, and (D) Opalescence Boost plus Nd:YAG laser treatment. Samples were then treated according to the following protocols (Tables 1 and 2).

(a) Control. The group was stored in normal saline and no bleaching was performed

(b) Opalescence Boost Only. The samples received a conventional bleaching regimen, which included a onetime application of $40 \%$ hydrogen peroxide with a gel thickness of $1.5 \mathrm{~mm}$, remaining on the teeth for 15 minutes following manufacturer's instructions

(c) Opalescence Boost plus Er:YAG Laser Treatment. The samples were painted with the whitening gel and irradiated with a $2940 \mathrm{~nm}$ Er:YAG laser (LightWalker, Fotona, Slovenia). The gel was activated with a bleaching setting of $0.4 \mathrm{w}$ and $10 \mathrm{~Hz}$ in VLP mode (1000microsecond pulse duration) using the R17 handpiece for 20 seconds ( 20 seconds per enamel specimen) at a $2 \mathrm{~cm}$ working distance and $5 \mathrm{~mm}$ spot size diameter. The gel was maintained on the specimens for 8 minutes after laser irritation. Finally, each specimen was rinsed with $5 \mathrm{~mL}$ sterile saline for 30 seconds

(d) Opalescence Boost plus Nd:YAG Laser Treatment. The tooth whitening gel was used in combination with a $1064 \mathrm{~nm} \mathrm{Nd:YAG} \mathrm{laser} \mathrm{(LightWalker,} \mathrm{Fotona,}$ Slovenia). The gel was activated with a bleaching setting of $8 \mathrm{w}$ and $60 \mathrm{~Hz}$ in VLP mode using the R24 handpiece five times for 4 seconds each time (20 seconds per enamel specimen) at a $2 \mathrm{~cm}$ working distance and $6 \mathrm{~mm}$ spot size diameter. The gel was maintained on the specimens for 8 minutes after laser irradiation. Finally, each specimen was rinsed with $5 \mathrm{~mL}$ sterile saline for 30 seconds

2.2. Scanning Electron Microscopy. Three samples from each group were examined under SEM (Zeiss, Germany). Samples were fixed with $2.5 \%$ glutaraldehyde and then dehydrated 
TABLE 1: Bleaching program in different treatment groups.

\begin{tabular}{lcccc}
\hline Group & Bleaching gel & Bleaching time & Er:YAG laser & Nd:YAG laser \\
\hline Control & - & 0 & - & - \\
Opalescence Boost only & Opalescence Boost 40\%PF & $15 \mathrm{~min}$ & - & - \\
Opalescence Boost plus Er: YAG laser & Opalescence Boost 40\%PF & $8 \mathrm{~min}$ & $20 \mathrm{~s}$ & - \\
Opalescence Boost plus Nd:YAG laser & Opalescence Boost 40\%PF & $8 \mathrm{~min}$ & - & $5 * 4 \mathrm{~s}$ \\
\hline
\end{tabular}

TABLE 2: Fotona dual-wavelength laser used for teeth whitening data.

\begin{tabular}{lcccccc}
\hline Program & Laser source & Pulse width & Frequency & Spot diameter & Power & Hand tools \\
\hline Bleaching & Er:YAG laser & VLP & $10 \mathrm{~Hz}$ & $5 \mathrm{~mm}$ & $0.4 \mathrm{~W}$ & R17 \\
Bleaching & Nd:YAG laser & VLP & $60 \mathrm{~Hz}$ & $6 \mathrm{~mm}$ & $8 \mathrm{~W}$ & R24 \\
\hline
\end{tabular}

successively with $30 \%, 50 \%, 70 \%$, 95\%, and 100\% ethanol. Then, the samples were dried at a critical point. Photomicrographs were obtained using different magnifications up to $5,000 \times$ to detect any changes in surface morphology.

2.3. Bacterial Cultures. Frozen $\left(-80^{\circ} \mathrm{C}\right)$ stocks of Streptococcus mutans (Sm) UA159, Streptococcus sanguis (Ss) ATCC10556, Actinomyces viscosus (Av) ATCC19246, and Fusobacterium nucleatum (Fn) ATCC25586 (provided by the Oral Microbiology Laboratory, The Ninth People's Hospital, Shanghai Jiao Tong University School of Medicine) were resuspended, transferred onto Brain heart infusion agar plates, and incubated at $37^{\circ} \mathrm{C}$ with $5 \% \mathrm{CO}_{2}$ for 48 hours. A single colony was then inoculated into sterile brain heart infusion media and incubated at $37^{\circ} \mathrm{C}$ with $5 \% \mathrm{CO}_{2}$ for $16 \mathrm{~h}$. Then, $50 \mu \mathrm{L}$ of each bacterial solution was obtained, Gram-stained, and assessed under an optical microscope (Nikon, Japan) to verify that the bacteria were free of contamination. The optical densities of each bacterial suspension were measured at $550 \mathrm{~nm}\left(\mathrm{OD}_{550 \mathrm{~nm}}\right)$ with a spectrophotometer, and the bacterial concentration was adjusted to about $10^{5-6} \mathrm{CFU} / \mathrm{mL}$. The four bacterial suspensions were then mixed in equal volumes in brain heart infusion containing $1 \%$ sucrose to perform biofilm assays.

2.4. Biofilm Formation Assays. The sterilized specimens were added to 24 -well plates and $400 \mu \mathrm{L}$ of bacterial suspensions containing $1 \%$ sucrose in BHI was dispensed onto each enamel sample. For the negative control, each group had a well that received media only, without bacteria or enamel. All samples were incubated at $37^{\circ} \mathrm{C}$ with $5 \% \mathrm{CO}_{2}$ for 24 or 48 hours.

The total amount of biofilm formed ( $n=4$ for each group) was quantified using crystal violet. The bound crystal violet was extracted using $400 \mu \mathrm{L}$ of destaining solution $(95 \%$ ethanol). A volume of $200 \mu \mathrm{L}$ was then transferred to a new 96-well plate. A microplate reader (Molecular Devices, USA) was used to measure the optical density of the destaining solution at $595 \mathrm{~nm}\left(\mathrm{OD}_{595 \mathrm{~nm}}\right)$, which represented the amount of total biofilm formed. The mean optical densities of the background groups were removed from the optical density values of the crystal violet values from their respective biofilm groups.

An additional three specimens from each group were prepared with SYTO9/PI fluorescent stain (Sigma, USA) and incubated for 15 minutes in the dark. Then, the biofilm was observed using a laser confocal scanning microscope (LSM510, Zeiss, Germany). The observation conditions included an Ar laser at $488 \mathrm{~nm}$, an HeNe laser at $543 \mathrm{~nm}$, and a $63 \times$ oil lens. Three different sites were randomly observed on each enamel sheet. Finally, to measure the thickness of the biofilm after 48 hours.

2.5. Statistical Analysis. Statistical calculations were performed using SPSS version 26.0 software (SPSS Inc., Chicago, IL, USA). The amount of biofilm formed between different groups was evaluated using the Kruskal-Wallis test. Statistical significance level was at $\alpha=0.05$.

\section{Results}

3.1. SEM Observations. The SEM observations were recorded to compare the structure between the groups at a magnification of up to $5,000 \times$ (Figure 1). Scratches caused by strong polishing were observed at $1,000 \times$ magnification. At $5,000 \times$ magnification, the surface of the control samples revealed no signs of enamel damage, while bleached enamels showed varying degrees of surface alterations, including porosities and cracks. The surfaces that underwent bleaching only showed partial damage with notable enamel dissolution and the presence of tomes processes. Unlike the bleached only, samples that underwent Er:YAG laser-activated bleaching exhibited noticeable losses in the integrity of the enamel surface. Additionally, enamel erosion was also indicated by the shearing of enamel rods. In those samples, the interprismatic spaces were predominantly damaged, and the loss of the interprismatic substance was evident. In the samples treated with $\mathrm{Nd}$ :YAG, the loss of integrity of the enamel was more aggressive than control, and surface fractures were observed.

3.2. Biofilm Formation. From biofilm formation assays, the OD values of mixed biofilms from all four groups after 24 hours were low, and no differences were observed between 
A
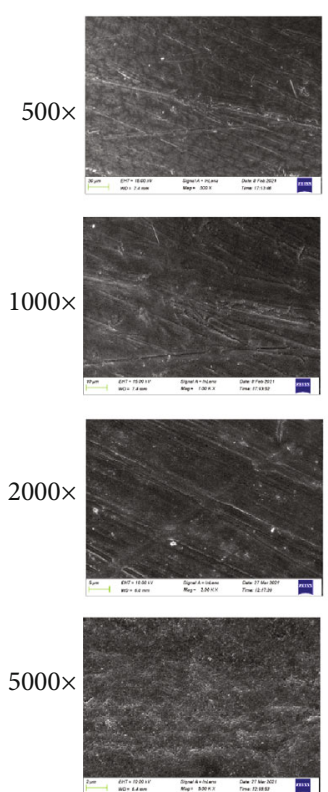

B
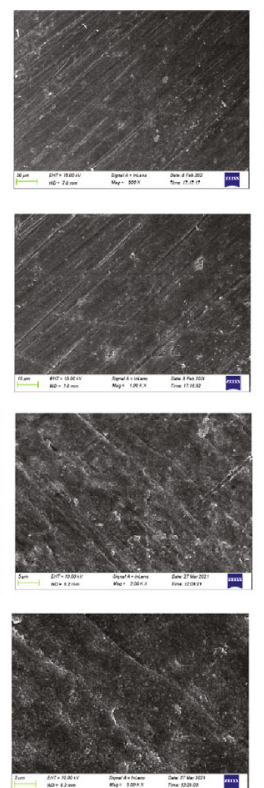

$\mathrm{C}$
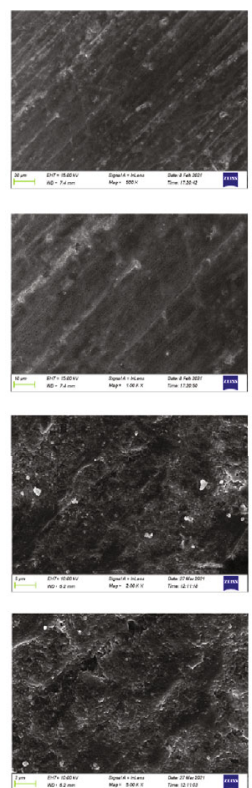

$\mathrm{D}$
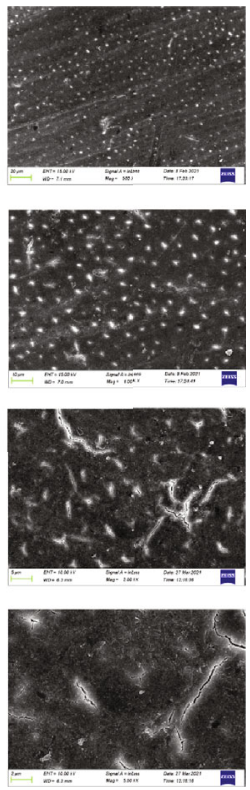

FIgURe 1: Scanning electron microscopy (SEM) images showing the structure of enamel surfaces (magnification up to 5,000×). (a) Control; (b) Opalescence Boost only; (c) Opalescence Boost with Er:YAG laser; (d) Opalescence Boost with Nd:YAG laser.
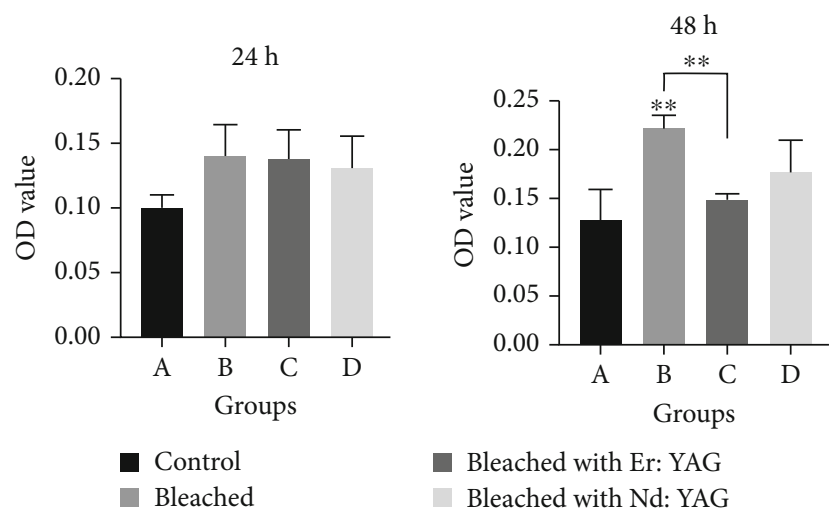

FIGURE 2: Crystal violet assays with optical density values at $595 \mathrm{~nm}$ (OD595 nm) representing the amount of mixed biofilms on the enamel. The error bars represent the standard deviation of measurements for 4 samples in each group. Asterisk $(*)$ represents significant differences $(p<0.05)$ according to KruskalWallis tests. (a) Control; (b) Opalescence Boost only; (c) Opalescence Boost with Er:YAG laser; (d) Opalescence Boost with Nd:YAG laser.

groups. However, after 48 hours, the OD values increased and the levels of biofilm formation on the enamel samples were significantly different. Bleaching only using $40 \%$ $\mathrm{H}_{2} \mathrm{O}_{2}$ led to the highest level of biofilm formation, while there was no significant difference in biofilm formation between the other groups (Figure 2).

The CLSM images (Figure 3) revealed the structures of biofilms in each treatment group, with the bacteria being stained green (live bacteria) or red (dead bacteria). For the 24 hours' biofilm, similar structures and thin biofilms were observed on the enamel surfaces in all groups. For 48 hours' biofilms, the number of cells in the unbleached group was lower than that of the bleached groups, while the bleached only group contained the most bacteria. Similar biofilm structures were observed on the enamel surfaces of samples bleached and treated with Er:YAG or Nd:YAG laser. And the bleached only group had the thickest biofilm after 48 hours (Table 3), which is consistent with the results observed by confocal microscopy.

\section{Discussion}

With the increased popularity of dental bleaching and the introduction of newer bleaching approaches, concerns have been raised about the efficacy, side effects, and risk of caries associated with such approaches [24]. Therefore, the aim of this study was to evaluate the enamel surface structures and biofilm formation after tooth bleaching using two new types of laser-activated approaches.

On the basis of the results reported in the current study, Hol stating that the laser-assisted tooth bleaching treatments would not change the enamel structure compared to the conventional bleaching technique was rejected. Our results demonstrated that the surface of the control samples revealed no evidence of enamel damage, while bleached enamel samples exhibited varying degrees of surface changes, including increased porosities and cracks.

Studies have shown that the structure and morphology of enamel can be affected by bleaching with laser irradiation [25]; however, the findings of such studies have not been consistent $[4,12,14,26,27]$. In our study, partial exposure of enamel rods after bleaching with Er:YAG laser was similar to the finding bleached with Er:YAG laser with 6\% or $35 \% \mathrm{H}_{2} \mathrm{O}_{2}$ [8]. Er:YAG laser cannot penetrate into enamel 


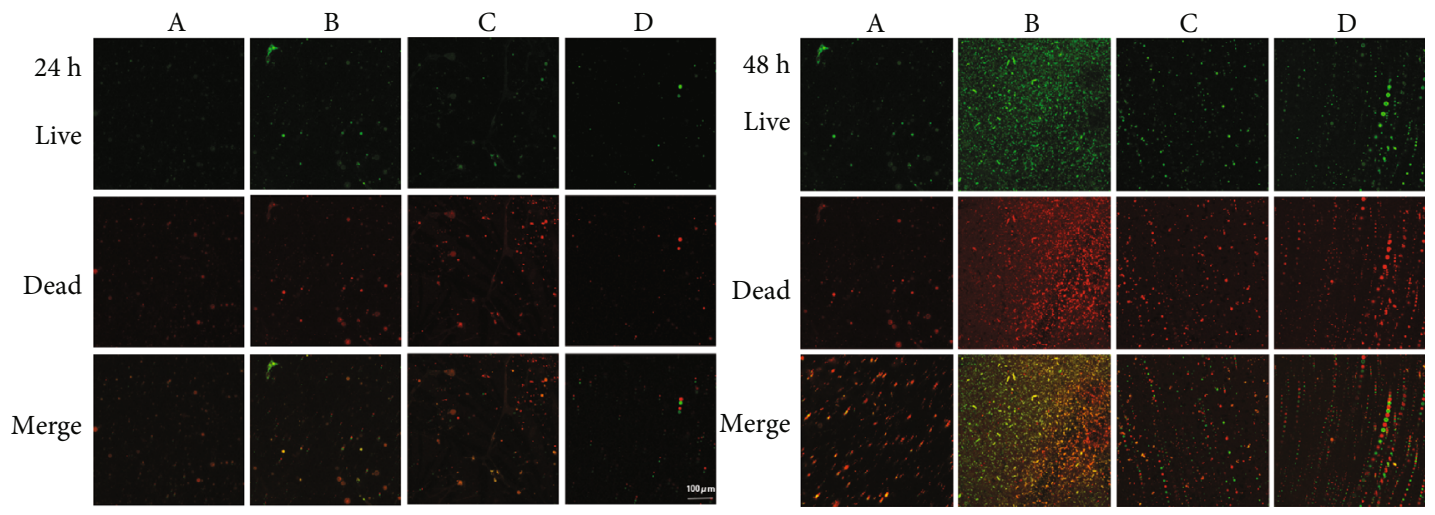

Figure 3: Confocal laser scanning microscope (CLSM) images showing the structure of biofilms on enamel surfaces (63×). (a) Control; (b) Opalescence Boost only; (c) Opalescence Boost with Er:YAG laser; (d) Opalescence Boost with Nd:YAG laser.

TABLE 3: Biofilm thickness in each group after 48 hours, $n=3$.

\begin{tabular}{lcccc}
\hline Group & A & B & C & D \\
\hline Biofilm thickness $(\mu \mathrm{m})$ & $7.67 \pm 3.21$ & $43.3 \pm 2.89$ & $12 \pm 1$ & $14.67 \pm 3.05$ \\
\hline
\end{tabular}

deeply, but can cause microblasting of inorganic substances and ablation of organic substances, so the enamel becomes molten and the enamel rods were exposed. Surface fracture was observed in the group receiving $\mathrm{Nd}$ :YAG laser treatment because Nd:YAG laser can quickly penetrate into the tooth and destroy enamel. But other studies suggested that the surface treated by Er:YAG laser showed irregular and microporous surface with flake pattern [13], and the surfaces treated with Nd:YAG lasers exhibited some melting and recrystallized areas [11]. Such differences could be due to the variations in different conditions, such as the tooth substrates (human or bovine), concentration and $\mathrm{pH}$ of bleaching gel, laser wavelength, and irradiation time, among other variables. However, it is unclear how enamel surface changes affect biofilm formation or other clinical parameters after bleaching using lasers.

Dental plaque biofilm refers to a clump of bacteria that accumulates on the tooth surface or other hard tissues and cannot be washed away by moderate water, and the dysbiosis of dental biofilms adhering to tooth surfaces can lead to caries [28]. It is well known that plaque biofilms rapidly formed within 24 hours and mature within 48 hours after the tooth surface was cleaned. Therefore, this study simulated the clinical procedures recommended by the manufactures and tested for the formation of mixed biofilms after 24 hours and 48 hours of culture.

At 24 hours, the OD values were relatively low, there was no difference between each group, and CLSM revealed a high proportion of dead bacteria due to the bactericidal effect of hydrogen peroxide, so the $\mathrm{H}_{0} 2$ was accepted. While $\mathrm{H}_{0} 3$ was rejected and after 48 hours of culture, an increase in biofilm formation was found on bleached enamel samples compared to the control samples. Additionally, samples treated by bleaching only with $40 \% \mathrm{H}_{2} \mathrm{O}_{2}$ led to the highest levels of biofilm formation, which was consistent with the report that bleaching leads to increased microbial adhesion on the enamel surface [29]. The increased attachment on bleached enamel may be due to the porosities and cracks on the enamel surface. However, biofilm formation on enamel bleached using lasers was lower than that on enamel bleached only, suggesting that laser bleaching has bactericidal or antibacterial effects. Although previous studies also suggested that laser irradiation may have a bactericidal effect $[30,31]$, it is not clear whether such photochemical toxicity exists when biofilm formation is carried out following laser irradiation. Thus, it is necessary to further evaluate the changes to enamel surface roughness and mineral content following laser treatment.

As the purpose of this study was to compare the effects of Er:YAG and Nd:YAG laser-activated bleaching systems, both laser sources were used with bleaching agents containing $40 \% \mathrm{H}_{2} \mathrm{O}_{2}$ to eliminate any discrepancies caused by the bleaching gels. In addition, because the strains and culture conditions used in this study were different from those in oral environments, the process, time, and structure of biofilms were different than those found under real circumstances. Therefore, additional experimental techniques and methods are needed to further reveal the effects of bleaching using different lasers on the formation of plaques on enamel surfaces.

\section{Conclusions}

The enamel surface structure significantly changed after bleaching with or without laser treatment. Bleaching only appeared to markedly promote biofilm formation after 48 hours, while biofilms formed on samples that underwent bleaching with Er:YAG or Nd:YAG laser did not change significantly, suggesting that bleaching with Er:YAG or 
Nd:YAG laser failed to promote the occurrence of caries and can be safely applied in clinical practice.

\section{Data Availability}

The figures data used to support the findings of this study are included within the article.

\section{Conflicts of Interest}

The authors do not have any financial interest in the companies whose materials are included in this article.

\section{Authors' Contributions}

Xiuxiu Hou did the conceptualization, methodology, validation, formal analysis, investigation, data curation, and writing-original draft. Keyong Yuan did the conceptualization, methodology, data curation, and writing-original draft. Zhengwei Huang did the conceptualization, methodology, formal analysis, and writing-review and editing. Rui $\mathrm{Ma}$ did the conceptualization, writing-review and editing, resources, supervision, project administration, and funding acquisition. All authors read and approved the final manuscript.

\section{Acknowledgments}

We thank International Science Editing for editing this manuscript. This work was supported by the Research Discipline fund no. KQYJXK2020 from Ninth People's Hospital, Shanghai Jiao Tong University School of Medicine, and College of Stomatology, Shanghai Jiao Tong University.

\section{References}

[1] Y. Gao, Z. Zhao, L. Li, K. Zhang, and Q. Liu, "In vitro evaluation of the effectiveness of bleaching agents activated by KTP and Nd:YAG laser," Photodiagnosis and Photodynamic Therapy, vol. 31, article 101900, 2020.

[2] M. Kashima-Tanaka, Y. Tsujimoto, K. Kawamoto, N. Senda, K. Ito, and M. Yamazaki, "Generation of free radicals and/or active oxygen by light or laser irradiation of hydrogen peroxide or sodium hypochlorite," Journal of Endodontia, vol. 29, no. 2, pp. 141-143, 2003.

[3] A. Joiner, "The bleaching of teeth: a review of the literature," Journal of Dentistry, vol. 34, no. 7, pp. 412-419, 2006.

[4] M. Q. Alqahtani, "Tooth-bleaching procedures and their controversial effects: a literature review," The Saudi Dental Journal, vol. 26, no. 2, pp. 33-46, 2014.

[5] D. S. Coutinho, L. Silveira, R. A. Nicolau, F. Zanin, and A. Brugnera, "Comparison of temperature increase in in vitro human tooth pulp by different light sources in the dental whitening process," Lasers in Medical Science, vol. 24, no. 2, pp. 179-185, 2009.

[6] A. Strobl, N. Gutknecht, R. Franzen, R. D. Hilgers, F. Lampert, and J. Meister, "Laser-assisted in-office bleaching using a neodymium:yttrium-aluminum-garnet laser: an in vivo study," Lasers in Medical Science, vol. 25, no. 4, pp. 503-509, 2010.

[7] B. C. Saglam, M. M. Kocak, S. Kocak, S. A. Turker, and D. Arslan, "Comparison of Nd:YAG and diode laser irradia- tion during intracoronal bleaching with sodium perborate: color and Raman spectroscopy analysis," Photomedicine and Laser Surgery, vol. 33, no. 2, pp. 77-81, 2015.

[8] C. Nguyen, C. Augros, J. P. Rocca, G. Lagori, and C. Fornaini, "KTP and Er:YAG laser dental bleaching comparison: a spectrophotometric, thermal and morphologic analysis," Lasers in Medical Science, vol. 30, no. 8, pp. 2157-2164, 2015.

[9] M. Mirzaie, E. Yassini, S. Ganji, Z. Moradi, and N. Chiniforush, "A comparative study of enamel surface roughness after bleaching with diode laser and Nd: YAG laser," Journal of Lasers in Medical Sciences, vol. 7, no. 3, pp. 197-200, 2016.

[10] S. Alia, "Effect of diode laser office bleaching on mineral content and surface topography of enamel Surface: An SEM Study," Journal of Clinical Pediatric Dentistry, vol. 13, no. 5, pp. 481-485, 2020.

[11] D. Dionysopoulos, D. Strakas, E. Koliniotou-Koumpia, and E. Koumpia, "Effect of Er, Cr: YSGG laser irradiation on bovine enamel surface during in-office tooth bleaching ex vivo," Odontology, vol. 105, no. 3, pp. 320-328, 2017.

[12] J. Kinoshita, H. Jafarzadeh, A. Manabe, M. Nozawa, T. Uchida, and P. V. Abbott, "Effects of KTP laser bleaching on traumatized tooth enamel," Trauma Monthly, vol. 19, no. 2, article e18168, 2014.

[13] M. M. Bassir, M. B. Rezvani, N. Chiniforush, and Z. Moradi, "In vitro evaluation of the effect of different laser irradiations on the enamel surfaces of teeth treated with home bleach procedure," Journal of Lasers Medical Sciencs, vol. 4, no. 4, pp. 168-174, 2013.

[14] E. Coceska, E. Gjorgievska, N. J. Coleman et al., "Enamel alteration following tooth bleaching and remineralization," Journal of Microscopy, vol. 262, no. 3, pp. 232-244, 2016.

[15] S. Ittatirut, O. Matangkasombut, and P. Thanyasrisung, "Inoffice bleaching gel with 35\% hydrogen peroxide enhanced biofilm formation of early colonizing streptococci on human enamel," Journal of Dentistry, vol. 42, no. 11, pp. 1480-1486, 2014.

[16] S. Bianchi, G. Fantozzi, S. Bernardi, S. Antonouli, and M. A. Continenza, "Commercial oral hygiene products and implant collar surfaces: scanning electron microscopy observations," Canadian Journal of Dental Hygiene, vol. 54, no. 1, pp. 2631, 2020.

[17] L. Gao, T. Xu, G. Huang, S. Jiang, Y. Gu, and F. Chen, "Oral microbiomes: more and more importance in oral cavity and whole body," Protein \& Cell, vol. 9, no. 5, pp. 488500, 2018.

[18] A. Briso, U. Silva, M. Souza, V. Rahal, E. G. Jardim Junior, and L. Cintra, "A clinical, randomized study on the influence of dental whitening on Streptococcus mutans population," Australian Dental Journal, vol. 63, no. 1, pp. 94-98, 2018.

[19] N. Hosoya, K. Honda, F. Iino, and T. Arai, "Changes in enamel surface roughness and adhesion of_Streptococcus mutans_to enamel after vital bleaching," Journal of Dentistry, vol. 31, no. 8, pp. 543-548, 2003.

[20] W. Krzysciak, A. Jurczak, D. Koscielniak, B. Bystrowska, and A. Skalniak, "The virulence of Streptococcus mutans and the ability to form biofilms," European Journal of Clinical Microbiology \& Infectious Diseases, vol. 33, no. 4, pp. 499-515, 2014.

[21] B. Zhu, L. C. Macleod, T. Kitten, and P. Xu, "Streptococcus sanguinisbiofilm formation \& interaction with oral pathogens," Future Microbiology, vol. 13, no. 8, pp. 915-932, 2018. 
[22] L. Deng, W. Li, Y. He, J. Wu, B. Ren, and L. Zou, "Cross-kingdom interaction of_Candida albicans_and_Actinomyces viscosus_elevated cariogenic virulence," Archives of Oral Biology, vol. 100, pp. 106-112, 2019.

[23] I. Nedeljkovic, J. De Munck, A. A. Ungureanu et al., "Biofilminduced changes to the composite surface," Journal of Dentistry, vol. 63, pp. 36-43, 2017.

[24] E. Ergin, A. Ruya Yazici, B. Kalender et al., "In vitro comparison of an Er:YAG laser-activated bleaching system with different light-activated bleaching systems for color change, surface roughness, and enamel bond strength," Lasers in Medical Science, vol. 33, no. 9, pp. 1913-1918, 2018.

[25] R. J. De Moor, J. Verheyen, P. Verheyen et al., "Laser teeth bleaching: evaluation of eventual side effects on enamel and the pulp and the efficiency in vitro and in vivo," Scientific World Journal, vol. 2015, pp. 1-12, 2015.

[26] Y. Sa, L. Sun, Z. Wang et al., "Effects of two in-office bleaching agents with different $\mathrm{pH}$ on the structure of human enamel: an in situ and in vitro study," Operative Dentistry, vol. 38 , no. 1 , pp. 100-110, 2013.

[27] L. Sun, S. Liang, Y. Sa et al., "Surface alteration of human tooth enamel subjected to acidic and neutral $30 \%$ hydrogen peroxide," Journal of Dentistry, vol. 39, no. 10, pp. 686-692, 2011.

[28] L. Pang, Y. Wang, Y. Ye, Y. Zhou, Q. Zhi, and H. Lin, “Metagenomic analysis of dental plaque on pit and fissure sites with and without caries among adolescents," Frontiers in Cellular and Infection Microbiology, vol. 11, article 740981, 2021.

[29] H. Heshmat, M. H. Ganjkar, S. Jaberi, and M. J. K. Fard, "The effect of remin pro and MI paste plus on bleached enamel surface roughness," Journal of Dentistry, vol. 11, no. 2, pp. 131-136, 2014.

[30] H. S. AlMoharib, B. Steffensen, D. Zoukhri, M. Finkelman, and R. Gyurko, "Efficacy of an Er:YAG laser in the decontamination of dental implant surfaces: an in vitro study," Journal of Periodontology, vol. 92, no. 11, pp. 1613-1621, 2021.

[31] A. de Souza da Fonseca, L. P. da Silva Sergio, A. L. Mencalha, and F. de Paoli, "Low-power lasers on bacteria: stimulation, inhibition, or effectless?," Lasers in Medical Science, vol. 36, no. 9, pp. 1791-1805, 2021. 